\title{
Digital Back-propagation for Unrepeatered Transmission
}

\author{
Domaniç Lavery \\ Optical Networks Group, Dept. Electronic \& Electrical Engineering \\ UCL (University College London), London WC1E 7JE, UK \\ Email: d.lavery@ucl.ac.uk
}

\begin{abstract}
Unrepeatered transmission has seen a substantial advance in recent years, largely due to the development of advanced optical fibers and amplifier technologies. Here, the potential, limitations and practicalities of digital nonlinearity compensation to build on this development are explored.
\end{abstract}

\section{INTRODUCTION}

Unrepeatered transmission covers a wide range of optical communications systems, including optical access, submarine, and optoelectronically regenerated networks. The common goal in all such networks is to jointly increase the transmission distance and throughput.

In single span experiments to date, this has been approached, in part, by improving the transmission medium itself through low loss, large effective area/low nonlinearity optical fibers and/or hybrid fiber spans [1], [2]. However, the most significant strides in performance have been due to amplifier technology, including the development of rare earth doped fiber amplifiers (e.g., the erbium doped fiber amplifier (EDFA)), forward- and backward-pumped stimulated Raman amplification [3], higher order Raman amplification [4], ultralong Raman fiber lasers [5], and remotely pumped optical amplifiers (ROPA) [6], [7].

One final technology to note is the digital coherent receiver. The linear detection characteristic of phase- and polarizationdiverse coherent receivers allows the full optical field to be mapped to the digital domain, enabling spectrally efficient advanced modulation formats, such as dual polarization m-ary quadrature amplitude modulation (DP-mQAM). Digital signal processing (DSP) can then be applied to compensate linear channel impairments, such as polarization mode dispersion, chromatic dispersion, and laser phase noise. Additionally, digital coherent receivers can be used to apply digital nonlinearity compensation (NLC) to the received signal, providing further scope to improve transmission performance.

NLC algorithms have almost exclusively been applied to multi-span optical networks. For the relatively small set of published research exploring NLC for passive optical networks in general, the nonlinear equalization has mainly used either Volterra filtering [8], or a simplified digital back-propagation (DBP) implementation [9]; exploiting, in both cases, the limited channel memory of particularly short single span links.

Unrepeatered transmission systems can exhibit unusual signal power profiles due to both ROPA and Raman amplification. Typically, for EDFA-based systems, DBP is implemented using a monotonically decreasing power profile, as is usually seen in multi-span systems. Matching the true signal power profile in single span Raman-amplified systems is crucial if the full gain of DBP is to be achieved. In the last year, there have been two notable experimental demonstrations of a full field, multi-channel, DBP algorithm for ultra-long single span transmission systems which correctly meet this requirement.

The first is the recent, record-breaking work [2], which used multi-channel transmitter-side DBP to enable a Q-factor gain of $0.8 \mathrm{~dB}$. In conjunction with the optimised hybrid fiber span, Raman amplification and ROPA, this achieved a record $20.7 \mathrm{~Tb} / \mathrm{s}$ over $400 \mathrm{~km}$ (although the DBP gain was reduced for the high capacity demonstration). The second notable demonstration used forward- and backward-pumped Raman amplification and receiver-based DBP to achieve approximately $2 \mathrm{~dB}$ Q-factor gain [3].

Although transmitter-side DBP is theoretically optimum for single span transmission [9], [10], one must take account of the noise introduced by the transmitter and receiver to truly optimise nonlinearity compensation ${ }^{1}[11]$. This is possibly why, to date, superior gains have been shown using receiver-side DBP for single span. In the following section, the transmission system employing receiver-side DBP [3] is taken as a case study to explore digital NLC in unrepeatered links.

\section{DBP ALGORITHMS FOR RAMAN-AMPLIFIED SPANS}

Consider the single span transmission system shown in Fig. 1, comprising the transmission of $7 \times 10 \mathrm{GBd}$ Nyquistspaced DP-16QAM channels over a second-order forward- and backward-pumped Raman amplified span (details in [3]). A typical second order Raman signal power profile is shown in Fig. 2. In contrast to transmission without distributed amplification (fiber attenuation only, as also shown in Fig. 2), the signal power exhibits peaks near the beginning and end of the span, which must be modelled by DBP.

Incorporating this power profile into the standard DBP algorithm (as in [13]), and applying DBP to all 7 channels, the achievable information rate (AIR) of the central channel - here defined as the estimated mutual information using an additive Gaussian noise auxilliary channel [3] - increases as a function of the number of nonlinear steps used for DBP,

\footnotetext{
${ }^{1}$ In reality, dividing DBP between transmitter and receiver is likely to yield the best performance [12].
} 


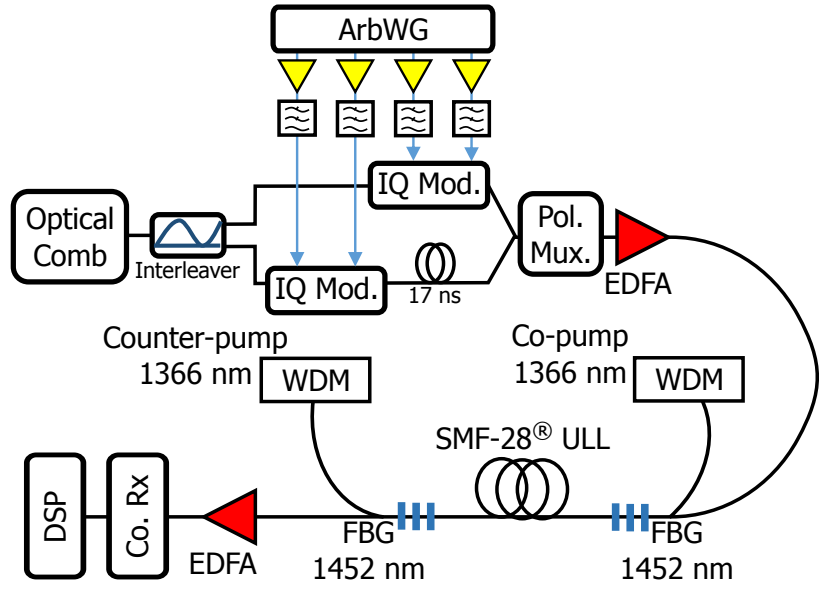

Fig. 1. The experimental configuration used to test the DBP algorithms. For details, see [3].

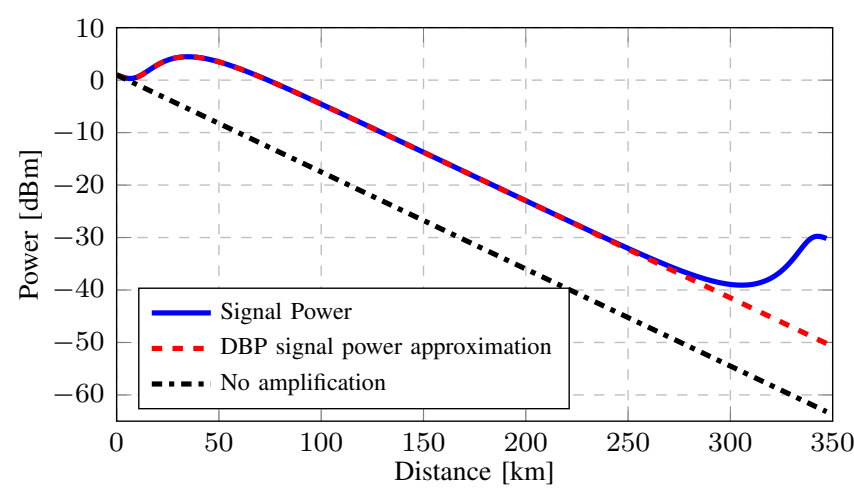

Fig. 2. Simulated signal power distribution over $347 \mathrm{~km}$ for the Raman amplified span, with a signal launch power of $1 \mathrm{dBm}$ per channel, and forward and backward pump powers of 30.9 and $31.4 \mathrm{dBm}$, respectively. The approximated power profile used for DBP is also shown alongside an unamplified span reference curve.

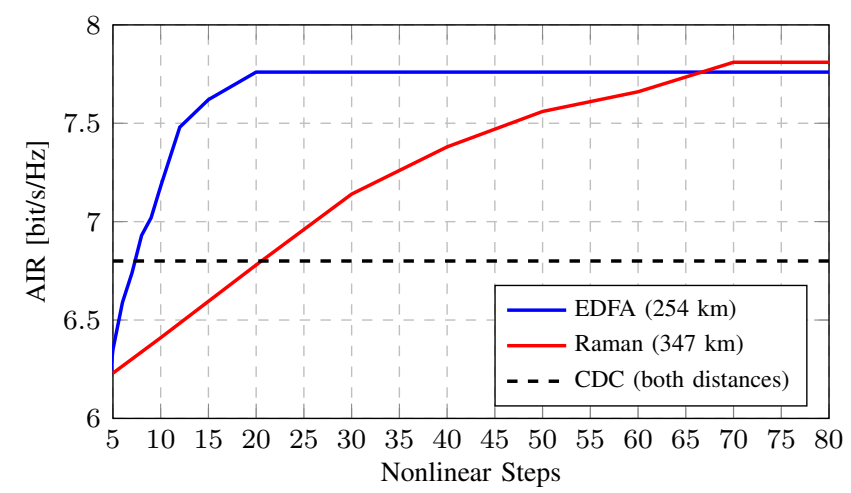

Fig. 3. Achievable rates (at optimum launch power) as a function of the number of DBP steps using EDFA and Raman systems. Assumes full field (70 GHz) DBP.

as shown in Fig. 3. Interestingly, due to the distributed gain and nonlinear interference, the Raman-amplified span requires a greater number of nonlinear steps to achieve optimum performance than the equivalent transmission system without Raman amplification (EDFA only).
From this it can be concluded that, although the performance gain afforded by DBP is comparable for both EDFAand Raman-amplified transmission spans, the complexity of a constant nonlinear step size implementation of DBP is significantly greater for Raman than EDFA, due to the fluctuating power profile. For EDFA-based systems, simplified, logarithmic step size DBP algorithms have been used to exploit the distribution of nonlinear interference throughout the span. For Raman-amplified spans, one approach to remedy this issue is to simplify the power profile, and then apply similar approximations. An approximated power profile is shown in Fig. 2, which achieves the same gain as the true power profile, and represents a first step towards a simplified DBP for unrepeatered systems.

\section{ACKNOWLEDGMENT}

The author thanks Dr. Lidia Galdino for her assistance with the preparation of this manuscript and enlightening discussions, and Prof. Polina Bayvel for her continued support. This work was supported by UK EPSRC Programme Grant UNLOC, EP/J017582/1, and the Royal Academy of Engineering under the Research Fellowships scheme.

\section{REFERENCES}

[1] J. D. Downie et al., "Quasi-Single-Mode Fiber Transmission for Optical Communications," IEEE J. Sel. Top. Quantum Electron., vol. 23, no. 3, pp. 1-12 (2017).

[2] Y. K. Huang et al., "20.7-Tb/s repeater-less transmission over 401.1-km using QSM fiber and XPM compensation via transmitter-side DBP,' in Proc. OptoElectronics and Communications Conference (OECC) (2016).

[3] L. Galdino et al., "Amplification Schemes and Multi-Channel DBP for Unrepeatered Transmission,” J. Lightwave Technol., vol. 34, no. 9, pp. 2221-2227 (2016).

[4] H. Bissessur et al., "24 Tb/s Unrepeatered C-Band Transmission of RealTime Processed 200 Gb/s PDM-16-QAM over 349 km," paper Th4D.2 in Proc. Optical Fiber Communication Conference (OFC) (2017).

[5] M. Tan et al., "Transmission comparison of ultra-long Raman fibre laser based amplification with first and dual order Raman amplification using $10 \times 118$ Gbit/s DP-QPSK," in Proc. International Conference on Transparent Optical Networks (ICTON) (2014).

[6] D. Chang et al., "Ultra-long unrepeatered transmission over $607 \mathrm{~km}$ at $100 \mathrm{G}$ and $632 \mathrm{~km}$ at 10G," Opt. Express, vol. 23, no. 19, pp. 2502825033 (2015).

[7] T. J. Xia et al., "557-km unrepeatered 100G transmission with commercial raman DWDM system, enhanced ROPA, and cabled large $\mathrm{A}_{\text {eff }}$ ultra-low loss fiber in OSP environment," paper Th5A.7 in Proc. Optical Fiber Communication Conference (OFC) (2014).

[8] H-Y. Chen et al., "Frequency- and time-domain nonlinear distortion compensation in high-speed OFDM-IMDD LR-PON with high loss budget," Opt. Express vol. 25, no. 5, pp. 5044-5056 (2017).

[9] D. Lavery et al. "Low Complexity Multichannel Nonlinear Predistortion for Passive Optical Networks", SpS2C.5 in Proc. Signal Processing in Photonics Communications (SPPCom) Conference (2015).

[10] E. Temprana et al., "Two-fold transmission reach enhancement enabled by transmitter-side digital backpropagation and optical frequency combderived information carriers," Opt. Express vol. 23, no. 16, pp. 2077420783 (2015)

[11] L. Galdino et al., "On the limits of digital back-propagation in the presence of transceiver noise" Opt. Express, vol. 25, no. 4, pp. 45644578 (2017).

[12] D. Lavery, et al. "The Benefit of Split Nonlinearity Compensation for Single-Channel Optical Fiber Communications," IEEE Photonics Technol. Lett., vol. 28, no. 17, pp. 1803-1806 (2016).

[13] L. Zhu and G. Li, "Impairment compensation for unrepeatered fiber transmission with distributed Raman amplification," in Proc. SPIE 8054, 80540S (2011). 